\title{
3 Research Suare \\ Fear of Childbirth and Depressive Symptoms Among Pregnant Women: A Cross-sectional Survey in Tanzania
}

Agnes Massae ( $\sim$ agysawe@yahoo.com )

Department of Women's and Children's Health, Uppsala University, Sweden

Agneta Svanberg

Uppsala University

Margareta Larsson

Uppsala University

Andrea Pembe

Muhimbili University of Health and Allied Sciences

Sebalda Leshabari

Muhimbili University of Health and Allied Sciences

Columba Mbekenga

Aga Khan University

\section{Research Article}

Keywords: Fear of Childbirth, Depressive symptoms, Pregnancy, Childbirth, Prevalence, Predictors, Tanzania

Posted Date: January 11th, 2021

DOI: https://doi.org/10.21203/rs.3.rs-124294/v1

License: (c) (i) This work is licensed under a Creative Commons Attribution 4.0 International License. Read Full License 


\section{Abstract}

Background: Many women experience fear of childbirth ( $F o B)$ and depressive symptoms (DS) during pregnancy, but little is known about FoB among Tanzanian women. The current study aimed to assess the prevalence of FoB and DS among pregnant women and determine predictors of each and both, focusing on sociodemographic and obstetric predictors.

Methods: A cross-sectional study was conducted at six health facilities in two districts in Tanzania between 2018 and 2019. In total, 694 pregnant women with gestational age between 32 and 40 weeks and expecting vaginal delivery were consecutively recruited and assessed for FoB and DS. We collected data through interviews using the Wijma Delivery Expectancy Questionnaire Version A and Edinburgh Postnatal Depression Scale. We performed multivariable logistic regression to investigate the predictors of FoB and DS.

Results: The prevalence rates of FoB and DS among pregnant women were $15.1 \%$ and $17.7 \%$, respectively. FoB and DS were more likely in women aged above 30 years (AOR 6.29, 95\% $\mathrm{Cl} 1.43-27.84$ ) and in single mothers (AOR 2.57, 95\% $\mathrm{Cl} 1.14-5.78$ ). Women with secondary education and above and those who had given birth previously were less likely to have FoB (AOR $0.22,95 \% \mathrm{Cl} 0.05-0.99$ ) and DS (AOR $0.27,95 \% \mathrm{Cl} 0.01-0.87$ ). Women who had previous obstetric complications, and those who did not receive any social support from male partners in previous childbirth were more likely to have FoB and DS. FoB was strongly associated with DS (AOR $3.42,95 \% \mathrm{Cl} 21.20-5.53$ ). DS only was more common in women who had inadequate income (AOR $2.35,95 \% \mathrm{Cl}$ 1.38-3.99) or had previously experienced a perineal tear (AOR $2.32,95 \% \mathrm{Cl} 1.31-4.08$ ).

Conclusions: Not having a formal education, having only primary education, being aged above 30 years, being single, being nulliparous, having experienced obstetric complications, and having a lack of social support from a male partner during previous pregnancy and childbirth were predictors of FoB and DS during pregnancy. FoB and DS were strongly associated with each other. It is vital to identify at-risk women early, to offer support during pregnancy and childbirth.

\section{What Is Already Known}

Nulliparous women report a higher level of fear of childbirth (FoB) than parous women. The Wijma Delivery Experience/Expectancy Questionnaire version A and Edinburgh Postnatal Depression Scale are the most frequently used tools for measuring FoB and DS, respectively, during pregnancy and have been validated worldwide.

\section{What this study adds}

1. This is the first study to investigate the prevalence and predictors of FoB in Tanzania.

2. Previous obstetric complications are the strongest predictor of both FoB and DS.

3. Lack of formal education, being aged above 30 years, being single, and being nulliparous are predictors of FoB in combination with DS.

\section{Introduction}


Women's childbirth experiences are multidimensional and unique to each woman, and can be positive, negative, or a mixture of both. The experience can be influenced by personal feelings, perceptions of personal performance [1], mental and physical health, and communication and interaction with health care providers [2, 3]. Moreover, type of childbirth, childbirth preferences regarding vaginal birth versus caeserean section $(C / S)[4,5]$, freedom in the expression of feelings related to childbirth, involvement in the birth experience, support from a partner and relatives [1, 2, 5], and other environmental factors, like lack of privacy during childbirth [1], can affect the childbirth experience. Some women who have a negative birth experience develop fear of having more children in the future $[6-8]$.

The prevalence of antenatal fear of childbirth (FoB) varies between countries. Worldwide, FoB ranges between 5 and $30 \%$ [9-14] and depressive symptoms (DS) affect 5-34\% of women during pregnancy [15-19]. In previous studies, there is a correlation between FoB, other anxiety problems, and DS $[20,21]$.

Quantitative studies across the world have shown that factors associated with FoB include sociodemographic factors like young maternal age, lack of social support from male partners, relatives and friends, unemployment, financial worries, and a history of abuse [22-24]. Obstetric factors like nulliparity and adverse obstetric events, such as a previous operative birth, may provoke childbirth fear [22, 25-27]. Also, not being mentally/physically prepared for giving birth, an expectation of unendurable pain, a feeling of loss of control during labour and birth, and fear of death have been deemed to predict FoB $[25,26]$. Inappropriate support from the birth team contributes to low childbirth confidence among women $[22,26]$.

In previous studies, depressive symptoms were associated with unwanted pregnancy [28, 29], young age/teenage pregnancy $[29,30]$, low levels of education, unemployment, and low prestigious employment [28, 31, 32]. Other factors reportedly associated with significant DS include inadequate social support (emotional, physical, and financial) and poor relationships with spouse/partner [19, 28, 30, 32]. These include negative marital relationships involving abuse, insufficient care, infidelity, polygamy, and conflicts on unwelcome sexual practices [19, 31, 33].

FoB is associated with pre-existing psychological problems like DS. An association between FoB and depression has been reported in a register study of 788,317 pregnant women in Finland, where depression was found to be the most potent risk factor for FoB, regardless of parity [34]. There are similar findings from other studies performed around the world [35-37].

Fear of childbirth may have consequences in the lives of women and their babies. It is associated with poor emotional and psychological health for women in general $[23,38]$. During childbirth, it can lead to $\mathrm{C} / \mathrm{S}$ on maternal request [6, 39-41], increased use of pharmacologic pain relief [38], prolonged labour [42], aggravated maternal and foetal distress [38], and increased obstetric interventions, like labour argumentation [39, 40]. Maternal FoB and DS can also affect the infant's future, potentially leading to decreased birth weight, delayed psychosocial development, reduced breastfeeding duration, hindered growth, severe malnutrition, more episodes of diarrhoea, and poor compliance with immunisation plans $[23,42,43]$. This might create problematic mother-newborn bonding, with subsequent attachment difficulties that may affect infant growth and development, including cognitive development [44-46].

To the best of our knowledge, no published studies have assessed FoB and its predictors among Tanzanian women, despite advocacy on integrated mental health in maternal and child health services in the health system and proven long-lasting consequences of maternal FoB and/or DS. Studies on the prevalence of FoB and DS, their 
predictors, effects, and management, have been performed mainly in European countries, with a few from India [47] and African countries [13,14]. In Tanzania, some studies have assessed DS during pregnancy, revealing a prevalence of $11.5 \%$ in the Kilimanjaro region, northern Tanzania [17], and 33.8\% in the Mwanza region [19]. Antenatal care relates to women's care during pregnancy, with the target being a healthy mother and child at end of pregnancy/birth. It is essential to increase understanding of FoB and DS during pregnancy to enhance the chances of providing the right support. Hence, the study's primary objective was to determine the prevalence of FoB and DS among pregnant women at ${ }^{3} 32$ weeks of gestation. The secondary objective was to investigate the predictors of FoB and DS, focusing on sociodemographic factors and previous obstetric experiences.

\section{Methods}

\section{Study design}

This was a cross-sectional study investigating the prevalence and predictors of FoB and DS among pregnant women in Tanzania.

\section{Study setting}

We carried out the study in the Mkuranga and Kisarawe districts in the Pwani region, Tanzania. The Mkuranga district has 56 health facilities in total: 1 hospital, five health centres ( 2 governmental and three privately owned), 50 dispensaries (38 governmental and 12 privately owned). The Kisarawe district has a total of 36 health facilities, including 1 district hospital, 3 health centres, and 31 dispensaries (26 are governmental and five privately owned). In each district, one district hospital and two health centres, all of which were government-owned, were selected for the study. The selected health facilities had a volume of at least ten antenatal visits per day, and the district hospitals had facilities for childbirth by both vaginal delivery and C/S. In Tanzania, antenatal care is accessible in every public health care facility at no charge. A pregnant woman is recommended to make at least eight antenatal visits at these facilities prior to delivery; at these facilities where nurse-midwives are the primary care providers. In case of any complications identified when providing services, the nurse-midwife refers the woman to a specialist or higher-level health facility for further management.

\section{Study participants and procedure}

We recruited and interviewed a consecutive sample of pregnant women seeking antenatal services at selected health facilities from September 2018 to March 2019. The eligibility criteria were: being pregnant in at least the $32^{\text {nd }}$ week of gestation; speaking Kiswahili; being resident in the selected district; having had no previous $\mathrm{C} / \mathrm{S}$; anticipating vaginal birth; attending the relevant health facility for antenatal care. Of the 1,130 pregnant women approached, 702 were invited to the study, 8 refused participation, and 694 provided survey data. Women who refused to participate did so due to lack of time, having other commitments after receiving antenatal care or that their male partners were waiting for them; some women felt uncomfortable taking part in the survey.

We trained six research assistants (RAs) in the data collection tools, use of a visual scale, study participant recruitment, and data collection procedures, including ethical principles in data collection. During the actual data collection, RAs recruited study participants, obtained informed consent, and performed data collection through face-to-face interviews in the selected antenatal clinics. Interviews were selected as the data collection method, to maintain data collection consistency, overcome the illiteracy challenge, and enhance comprehension of the 
questions and data collection scales. To decrease interview bias, participants could use a visual analogue scale to rate levels of fear for the Wijma Delivery and Expectation Questionnaire version A (W-DEQ-A). This reduced the risk of having different verbal explanations for response items from different research assistants. To enhance validity, all interviews were conducted in Kiswahili.

\section{Instruments and measures}

The participants were interviewed regarding demographic and obstetric characteristics and their feelings and expectations of childbirth, using the W-DEQ-A [48]. They were also assessed on DS using the Edinburgh Postnatal Depression Scale (EPDS) [49]. Questions on obstetrics and social support were asked in relation to the history of the most recent previous pregnancy and childbirth.

The W-DEQ-A is a 6-point Likert scale questionnaire with 33 items ranging from 0 (not at all) to 5 (extremely), yielding a minimum score of 0 and a maximum score of 165 . The higher the score, the more intense the FoB. The cut-off points from the original tool are as follows: a score of $£ 37$ is considered a low level of fear, a score of 3865 reflects a moderate level of fear, a score of 66-84 signifies a high level of fear, and a score of ${ }^{3} 85$ indicates a severe level of fear [50]. In this study, we defined FoB as scoring ${ }^{3} 66$ and no FoB as scoring $<66$. The original WDEQ-A has been reported to be a reliable questionnaire with a Cronbach's alpha of 0.93 . The original English questionnaire version was translated into Kiswahili and reviewed by a team of native Kiswahili speakers and professionals in midwifery, obstetrics, behavioral science, psychology, and psychiatry. Further, a pilot study and validation process were carried out and found that the translated W-DEQ-A was a reliable tool, with a Cronbach's alpha of 0.83 [51] (unpublished, submitted).

The EPDS is a tool used to identify common perinatal mental disorders and has been used to screen women at risk of developing depression during pregnancy or/and after childbirth. The EPDS is a four-point Likert scale with 10 items ranging from 0 to 3 . Participants rate each item based on how they have felt in the preceding seven days. The lowest score that can be obtained on this scale is 0 , while the highest is 30 . The higher the score, the higher the risk of having DS. Scores ${ }^{3} 10$ suggest the presence of DS, while scores ${ }^{3} 13$ represent depressive illness of varying severity [49]. In this study, we defined DS as scoring ${ }^{3} 10$ and no DS as scoring $<10$. he internal consistency reliability of the original EPDS was excellent, with a Cronbach's alpha of 0.88 . Our study had a Cronbach's alpha of 0.85 . The EPDS has been translated into Kiswahili in Kenya and showed an acceptable Cronbach's alpha of 0.78 [18]. We adapted the EPDS from Kenya, translated by Green and colleagues [18], for data collection in Tanzania.

\section{Data analysis}

Data were analysed using the Statistical Package for the Social Sciences (SPSS) version 26. Prior to analysis, the normality test was performed for the total scores of FoB and DS using the Q-Q plot, histogram, box plot, and Kolmogorov-Smirnov. Descriptive statistics were used to summarise demographics, obstetric characteristics, and the prevalence rates of FoB and DS. A chi-squared test was performed to determine the associations between sociodemographic and obstetric variables and the prevalence rates of FoB and DS. Logistic regression was used to estimate the effects of sociodemographic and obstetric variables on the prevalence rates of FoB and DS after adjusting for potential confounders. Univariable analysis was conducted to assess the association between each sociodemographic and obstetric variable and the presence of FoB and/or DS. All the variables with $p<0.2$ in the univariable analysis were subjected to several multivariable logistic regression models using a hierarchical 
approach. A p value of < 0.05 was considered significant. Effect sizes were presented using odds ratios (ORs) with $95 \%$ confidence intervals (Cls). The outcomes of interest were grouped as (1) FoB vs. no FoB and DS vs. no DS, (2) Neither FoB nor DS vs. FoB but no DS, DS but no FoB, and FoB in combination with DS.

\section{Results}

\section{Description of the population}

In total, 694 eligible pregnant women agreed to participate in this study. Their age varied between 14 and 46 years, with a median age of 26 years, and the majority (73.2\%) were married and had primary education (61.8\%). The majority were financially and socially supported by their male partners and had planned the pregnancy.

\section{Prevalence of FoB and DS}

The total scores for FoB ranged from 1 to 102, while those for DS were $0-27$. The median scores with interquartile range (IR) for FoB and DS were 48 (24.0) and 3 (7), respectively. The overall prevalence of FoB was $15.1 \%$ and that of DS was $17.7 \%$. The results indicated that about 37 (5.3\%) of the pregnant women had both FoB and DS, 68 (9.8\%) had FoB but no DS, 86 (12.4\%) had DS but no FoB, and 503 (72.5\%) had neither FoB nor DS.

\section{Scores for the item in the W-DEQ-A}

Scores for each item in the W-DEQ-A are shown in Table 1 in descending order of prevalence. The most common fear reported by pregnant women was labour pain. The items for which most women scored low were longing for a child and childbirth being fun. 
Table 1: FoB scores by item.

\begin{tabular}{|c|c|c|c|}
\hline Item number & Items & Mean (SD) & Total \\
\hline 24 & Painful & $4.2(0.9)$ & 2918 \\
\hline 25 & Behave badly & $3.1(1.6)$ & 2147 \\
\hline 2 & Frightful & $2.8(1.6)$ & 1955 \\
\hline 3 & Lonely & $2.8(1.6)$ & 1913 \\
\hline 6 & Afraid & $2.7(1.6)$ & 1883 \\
\hline 19 & Panic & $2.7(1.5)$ & 1884 \\
\hline 26 & Surrender control of the body & $2.4(1.7)$ & 1651 \\
\hline 12 & Tense & $2.5(1.5)$ & 1768 \\
\hline 8 & Weak & $2.3(1.5)$ & 1616 \\
\hline 31 & Dangerous & $2.1(1.6)$ & 1446 \\
\hline 27 & Lose control & $1.8(1.4)$ & 1269 \\
\hline 7 & Deserted & $1.7(1.4)$ & 1213 \\
\hline 15 & Abandoned & $1.7(1.5)$ & 1198 \\
\hline 11 & Desolate & $1.6(1.4)$ & 1114 \\
\hline 1 & Fantastic & $1.5(1.5)$ & 1019 \\
\hline 13 & Glad & $1.5(1.4)$ & 1077 \\
\hline 20 & Hopelessness & $1.5(1.6)$ & 1066 \\
\hline 18 & Happy & $0.9(1.2)$ & 603 \\
\hline 17 & Relaxed & $0.8(1.1)$ & 549 \\
\hline 32 & Fantasy that child will die & $0.8(1.1)$ & 551 \\
\hline 4 & Strong & $0.7(0.9)$ & 478 \\
\hline 9 & Safe & $0.7(1.0)$ & 480 \\
\hline 10 & Independent & $0.7(1.1)$ & 507 \\
\hline 14 & Proud & $0.7(1.1)$ & 458 \\
\hline 16 & Composed & $0.7(1.0)$ & 506 \\
\hline 33 & Fantasy that child will be injured & $0.7(0.9)$ & 453 \\
\hline 5 & Confident & $0.6(0.9)$ & 399 \\
\hline 29 & Natural & $0.5(0.9)$ & 365 \\
\hline 30 & Self-evident & $0.5(1.0)$ & 344 \\
\hline
\end{tabular}




\begin{tabular}{|llll|}
\hline 22 & Self confidence & $0.4(0.8)$ & 289 \\
\hline 23 & Trust & $0.4(0.8)$ & 274 \\
\hline 21 & Longing for a child & $0.1(0.5)$ & 55 \\
\hline 28 & Funny & $0.1(0.5)$ & 73 \\
\hline Total FoB score & $\mathbf{4 8 . 2 ( 1 6 . 3 )}$ & $\mathbf{3 3 5 2 1}$ \\
\hline
\end{tabular}

\section{Association between demographic and obstetric variables and FoB and/or DS}

Education level, marital status, sex of the baby, male partner social support, and experience of obstetric complications were significant predictors of FoB $(p<0.05)$. DS and FoB were strongly associated $(p<0.001)$. DS was predicted by education level, marital status, income adequacy, previous perineal tear, and previous obstetric complication (Table 2).

Table 2: Associations of sociodemographic and obstetric variables with FoB and DS. 


\begin{tabular}{|c|c|c|c|c|c|c|c|}
\hline \multirow[b]{2}{*}{ Variables } & \multirow[b]{2}{*}{ Total } & \multicolumn{2}{|c|}{ FoB (W-DEQ-A) } & \multicolumn{4}{|c|}{ DS (EPDS) } \\
\hline & & $\begin{array}{l}\text { No fear } \\
(£ 65)\end{array}$ & $\begin{array}{c}\text { Fear } \\
\left(\begin{array}{l}3 \\
6\end{array} 6\right)\end{array}$ & $\begin{array}{l}\mathrm{P}- \\
\text { value }\end{array}$ & $\begin{array}{c}\text { No } \\
\text { DS (< } \\
10)\end{array}$ & $\begin{array}{l}\text { DS }{ }^{3} \\
10)\end{array}$ & $\begin{array}{l}\mathrm{P} \\
\text { value }\end{array}$ \\
\hline & $\mathrm{n}(\%)$ & $\mathrm{n}(\%)$ & n (\%) & & $\mathrm{n}(\%)$ & $\mathrm{n}(\%)$ & \\
\hline \multicolumn{8}{|c|}{$\begin{array}{l}\text { Index pregnancy variables ( } \\
=694 \text { ) }\end{array}$} \\
\hline Age group & & & & 0.371 & & & 0.235 \\
\hline$£ 20$ years & $\begin{array}{l}139 \\
(100)\end{array}$ & $\begin{array}{l}113 \\
(81.3)\end{array}$ & $\begin{array}{l}26 \\
(18.7)\end{array}$ & & $\begin{array}{l}118 \\
(84.9)\end{array}$ & $\begin{array}{l}21 \\
(15.1)\end{array}$ & \\
\hline $21-30$ years & $\begin{array}{l}358 \\
(100)\end{array}$ & $\begin{array}{l}305 \\
(85.2)\end{array}$ & $\begin{array}{l}53 \\
(14.8)\end{array}$ & & $\begin{array}{l}286 \\
(79.9)\end{array}$ & $\begin{array}{l}72 \\
(20.1)\end{array}$ & \\
\hline${ }^{3} 31$ years & $\begin{array}{l}197 \\
(100)\end{array}$ & $\begin{array}{l}171 \\
(86.8)\end{array}$ & $\begin{array}{l}26 \\
(13.2)\end{array}$ & & $\begin{array}{l}167 \\
(84.8)\end{array}$ & $\begin{array}{l}30 \\
(15.2)\end{array}$ & \\
\hline Education level & & & & 0.121 & & & 0.056 \\
\hline No formal education & $\begin{array}{l}137 \\
(100)\end{array}$ & $\begin{array}{l}116 \\
(84.7)\end{array}$ & $\begin{array}{l}21 \\
(15.3)\end{array}$ & & $\begin{array}{l}116 \\
(84.7)\end{array}$ & $\begin{array}{l}21 \\
(15.3)\end{array}$ & \\
\hline Primary education & $\begin{array}{l}429 \\
(100)\end{array}$ & $\begin{array}{l}357 \\
(83.2)\end{array}$ & $\begin{array}{l}72 \\
(16.8)\end{array}$ & & $\begin{array}{l}359 \\
(83.7)\end{array}$ & $\begin{array}{l}70 \\
(16.3)\end{array}$ & \\
\hline Secondary and higher & $\begin{array}{l}128 \\
(100)\end{array}$ & $\begin{array}{l}116 \\
(90.6)\end{array}$ & $\begin{array}{l}12 \\
(9.4)\end{array}$ & & $\begin{array}{l}96 \\
(75.0)\end{array}$ & $\begin{array}{l}32 \\
(25.0)\end{array}$ & \\
\hline Occupation & & & & 0.446 & & & 0.687 \\
\hline Employed & $\begin{array}{l}490 \\
(100)\end{array}$ & $\begin{array}{l}419 \\
(85.5)\end{array}$ & $\begin{array}{l}71 \\
(14.5)\end{array}$ & & $\begin{array}{l}405 \\
(82.7)\end{array}$ & $\begin{array}{l}85 \\
(17.3)\end{array}$ & \\
\hline Not employed & $\begin{array}{l}204 \\
(100)\end{array}$ & $\begin{array}{l}170 \\
(83.3)\end{array}$ & $\begin{array}{l}34 \\
(16.7)\end{array}$ & & $\begin{array}{l}166 \\
(81.4)\end{array}$ & $\begin{array}{l}38 \\
(18.6)\end{array}$ & \\
\hline Marital status & & & & 0.048 & & & 0.003 \\
\hline Married & $581(100)$ & $\begin{array}{l}500 \\
(86.1)\end{array}$ & $\begin{array}{l}81 \\
(13.9)\end{array}$ & & $\begin{array}{l}489 \\
(84.2)\end{array}$ & $\begin{array}{l}92 \\
(15.8)\end{array}$ & \\
\hline Single & $\begin{array}{l}113 \\
(100)\end{array}$ & $\begin{array}{l}89 \\
(78.8)\end{array}$ & $\begin{array}{l}24 \\
(21.2)\end{array}$ & & $\begin{array}{l}82 \\
(72.6)\end{array}$ & $\begin{array}{l}31 \\
(27.4)\end{array}$ & \\
\hline Income & & & & 0.286 & & & 0.001 \\
\hline Adequate & $\begin{array}{l}304 \\
(100)\end{array}$ & $\begin{array}{l}263 \\
(86.5)\end{array}$ & $\begin{array}{l}41 \\
(13.5)\end{array}$ & & $\begin{array}{l}267 \\
(87.8)\end{array}$ & $\begin{array}{l}37 \\
(12.2)\end{array}$ & \\
\hline Inadequate & $\begin{array}{l}390 \\
(100)\end{array}$ & $\begin{array}{l}326 \\
(83.6)\end{array}$ & $\begin{array}{l}64 \\
(16.4)\end{array}$ & & $\begin{array}{l}304 \\
(77.9)\end{array}$ & $\begin{array}{l}86 \\
(22.1)\end{array}$ & \\
\hline Gravidity & & & & 0.244 & & & 0.551 \\
\hline Primigravida & $\begin{array}{l}161 \\
(100)\end{array}$ & $\begin{array}{l}132 \\
(82.0)\end{array}$ & $\begin{array}{l}29 \\
(18.0)\end{array}$ & & $\begin{array}{l}135 \\
(23.6)\end{array}$ & $\begin{array}{l}26 \\
(21.1)\end{array}$ & \\
\hline
\end{tabular}




\begin{tabular}{|c|c|c|c|c|c|c|c|}
\hline Multigravida & $\begin{array}{l}533 \\
(100)\end{array}$ & $\begin{array}{l}457 \\
(85.7)\end{array}$ & $\begin{array}{l}76 \\
(14.3)\end{array}$ & & $\begin{array}{l}436 \\
(76.4)\end{array}$ & $\begin{array}{l}97 \\
(78.9)\end{array}$ & \\
\hline Parity & & & & 0.244 & & & 0.196 \\
\hline Nulliparous & $\begin{array}{l}347 \\
(100)\end{array}$ & $\begin{array}{l}289 \\
(83.3)\end{array}$ & $\begin{array}{l}58 \\
(16.7)\end{array}$ & & $\begin{array}{l}279 \\
(80.4)\end{array}$ & $\begin{array}{l}68 \\
(19.6)\end{array}$ & \\
\hline Parous & $\begin{array}{l}347 \\
(100)\end{array}$ & $\begin{array}{l}300 \\
(86.5)\end{array}$ & $\begin{array}{l}47 \\
(13.5)\end{array}$ & & $\begin{array}{l}292 \\
(84.1)\end{array}$ & $\begin{array}{l}55 \\
(15.9)\end{array}$ & \\
\hline Pregnancy status & & & & 0.693 & & & 0.165 \\
\hline Planned & $\begin{array}{l}471 \\
(100)\end{array}$ & $\begin{array}{l}398 \\
(84.5)\end{array}$ & $\begin{array}{l}73 \\
(15.5)\end{array}$ & & $\begin{array}{l}381 \\
(80.9)\end{array}$ & $\begin{array}{l}90 \\
(19.1)\end{array}$ & \\
\hline Unplanned & $\begin{array}{l}223 \\
(100)\end{array}$ & $\begin{array}{l}191 \\
(85.7)\end{array}$ & $\begin{array}{l}32 \\
(14.3)\end{array}$ & & $\begin{array}{l}190 \\
(85.2)\end{array}$ & $\begin{array}{l}33 \\
(14.8)\end{array}$ & \\
\hline DS during pregnancy & & & & $<0.001$ & & & \\
\hline No & $\begin{array}{l}571 \\
(100)\end{array}$ & $\begin{array}{l}503 \\
(88.1)\end{array}$ & $\begin{array}{l}68 \\
(11.9)\end{array}$ & & NA & & \\
\hline Yes & $\begin{array}{l}123 \\
(100)\end{array}$ & $\begin{array}{l}86 \\
(69.9)\end{array}$ & $\begin{array}{l}37 \\
(30.1)\end{array}$ & & NA & & \\
\hline FoB during pregnancy & & & & & & & $\hat{0.001}$ \\
\hline No & $\begin{array}{l}589 \\
(100)\end{array}$ & NA & & & $\begin{array}{l}503 \\
(85.4)\end{array}$ & $\begin{array}{l}86 \\
(14.6)\end{array}$ & \\
\hline Yes & $\begin{array}{l}105 \\
(100)\end{array}$ & NA & & & $\begin{array}{l}68 \\
(64.8)\end{array}$ & $\begin{array}{l}37 \\
(35.2)\end{array}$ & \\
\hline \multicolumn{8}{|l|}{$\begin{array}{l}\text { Previous birth and pregnancy } \\
\text { variables }(n=504)\end{array}$} \\
\hline $\begin{array}{l}\text { Social support from a male } \\
\text { partner in previous childbirth }\end{array}$ & & & & 0.016 & & & 0.112 \\
\hline Yes & $\begin{array}{l}287 \\
(56.9)\end{array}$ & $\begin{array}{l}236 \\
(82.2)\end{array}$ & $\begin{array}{l}51 \\
(17.8)\end{array}$ & & $\begin{array}{l}226 \\
(78.7)\end{array}$ & $\begin{array}{l}61 \\
(21.3)\end{array}$ & \\
\hline No & $\begin{array}{l}217 \\
(43.1)\end{array}$ & $\begin{array}{l}195 \\
(89.9)\end{array}$ & $\begin{array}{l}22 \\
(10.1)\end{array}$ & & $\begin{array}{l}183 \\
(84.3)\end{array}$ & $\begin{array}{l}34 \\
(15.7)\end{array}$ & \\
\hline Previous perineal tear & & & & 0.228 & & & 0.006 \\
\hline Yes & $\begin{array}{l}130 \\
(25.8)\end{array}$ & $\begin{array}{l}107 \\
(82.3)\end{array}$ & $\begin{array}{l}23 \\
(17.7)\end{array}$ & & $\begin{array}{l}95 \\
(73.1)\end{array}$ & $\begin{array}{l}35 \\
(26.9)\end{array}$ & \\
\hline No & $\begin{array}{l}374 \\
(74.2)\end{array}$ & $\begin{array}{l}324 \\
(86.6)\end{array}$ & $\begin{array}{l}50 \\
(13.4)\end{array}$ & & $\begin{array}{l}314 \\
(84.0)\end{array}$ & $\begin{array}{l}60 \\
(16.0)\end{array}$ & \\
\hline $\begin{array}{l}\text { Ever experienced obstetric } \\
\text { complications }\end{array}$ & & & & 0.007 & & & $<0.001$ \\
\hline
\end{tabular}




\begin{tabular}{|llllll|} 
Yes & 141 & 111 & 30 & 104 & 37 \\
& $(28.0)$ & $(78.7)$ & $(21.3)$ & $(73.8)$ & $(26.2)$ \\
\hline No & 363 & 320 & 43 & 305 & $53(16.0)$ \\
& $(72.0)$ & $(88.2)$ & $(11.8)$ & $(84.0)$ & \\
\hline
\end{tabular}

\section{Regression analysis of the predictors of FoB and DS}

Tables 3 and 4 show different logistic regression models for both univariable and multivariable analyses of FoB and DS as outcomes. The variables associated with FoB, DS, and both FoB and DS, were entered into multivariable analysis models, one after another. Age, education, and parity were included in every model, since they had been shown to be confounders of FoB and DS predictors in previous studies.

\section{Table 3: Demographic and obstetrics predictors of FoB and DS.}




\begin{tabular}{|c|c|c|c|c|}
\hline \multicolumn{3}{|l|}{ Fear of childbirth } & \multicolumn{2}{|c|}{ Depressive symptoms } \\
\hline \multicolumn{3}{|c|}{$n=105 / 694(15.1 \%)$} & \multicolumn{2}{|c|}{$n=123 / 694(17.7 \%)$} \\
\hline & Unadjusted & Adjusted & Unadjusted & Adjusted \\
\hline Variables & OR $(95 \% \mathrm{Cl})$ & OR $(95 \% \mathrm{Cl})$ & OR $(95 \% \mathrm{Cl})$ & OR (95\% Cl) \\
\hline \multicolumn{5}{|l|}{ Age } \\
\hline$\leq 20$ years & 1 & 1 & 1 & 1 \\
\hline $21-30$ years & $0.76(0.45-1.27)$ & $1.02(0.55-1.89)$ & $1.32(0.76-2.29)$ & $1.38(0.70-2.69)$ \\
\hline$\geq 31$ years & $0.66(0.37-1.19)$ & $0.88(0.38-2.03)$ & $0.93(0.49-1.73)$ & $1.05(0.44-2.53)$ \\
\hline \multicolumn{5}{|l|}{ Education level } \\
\hline No formal education & 1 & 1 & 1 & 1 \\
\hline Primary education & $1.11(0.66-1.89)$ & $0.94(0.54-1.64)$ & $1.08(0.63-1.83)$ & $0.90(0.51-1.61)$ \\
\hline $\begin{array}{l}\text { Secondary education and } \\
\text { above }\end{array}$ & $\begin{array}{l}0.58(0.27- \\
0.22)^{*}\end{array}$ & $\begin{array}{l}0.42(0.18- \\
0.94)^{*}\end{array}$ & $1.84(0.99-3.40)$ & $1.49(0.72-3.09)$ \\
\hline \multicolumn{5}{|l|}{ Parity } \\
\hline Nulliparous & 1 & 1 & 1 & 1 \\
\hline Parous & $0.78(0.51-1.19)$ & $0.78(0.42-1.45)$ & $0.77(0.52-1.14)$ & $0.79(0.43-1.44)$ \\
\hline \multicolumn{5}{|l|}{ Marital status } \\
\hline Married & 1 & 1 & 1 & 1 \\
\hline Single & $\begin{array}{l}2.01(1.33- \\
3.02)^{\star}\end{array}$ & $1.56(0.92-2.65)$ & $\begin{array}{l}2.01(1.33- \\
3.02)^{*}\end{array}$ & $\begin{array}{l}1.72(1.01- \\
2.91)^{*}\end{array}$ \\
\hline \multicolumn{5}{|l|}{ Planned pregnancy } \\
\hline No & 1 & & 1 & \\
\hline Yes & $1.09(0.69-1.72)$ & NA & $1.36(0.88-2.10)$ & NA \\
\hline \multicolumn{5}{|l|}{ Income } \\
\hline Adequate & & & 1 & 1 \\
\hline Inadequate & $1.26(0.82-1.93)$ & NA & $\begin{array}{l}2.04(1.34- \\
3.10)^{*}\end{array}$ & $\begin{array}{l}2.39(1.49- \\
3.83)^{\star \star}\end{array}$ \\
\hline \multicolumn{5}{|c|}{ Social support from a male partner in previous childbirth } \\
\hline No & 1 & 1 & 1 & 1 \\
\hline Yes & $0.75(0.49-1.14)$ & $\begin{array}{l}0.49(0.29- \\
0.79)^{*}\end{array}$ & $0.71(0.48-1.04)$ & $\begin{array}{l}0.61(0.37- \\
0.98)^{*}\end{array}$ \\
\hline \multicolumn{5}{|c|}{ Ever experienced obstetric complications } \\
\hline No & 1 & 1 & 1 & 1 \\
\hline Yes & $1.72(1.72-$ & $\begin{array}{l}2.03(1.24- \\
\text { age } 12 / 23\end{array}$ & $2.33(1.43-$ & $1.76(1.07-$ \\
\hline
\end{tabular}




\begin{tabular}{|c|c|c|c|c|}
\hline & $2.61)^{*}$ & $3.23) *$ & $3.81) *$ & $2.89) *$ \\
\hline \multicolumn{5}{|c|}{ Previous perineal tear } \\
\hline No & 1 & & 1 & 1 \\
\hline Yes & $1.26(0.76-2.09)$ & NA & $\begin{array}{l}2.03(1.26- \\
3.25)^{\star}\end{array}$ & $1.21(0.72-2.01)$ \\
\hline \multicolumn{5}{|l|}{ DS } \\
\hline No & 1 & & NA & NA \\
\hline Yes & $\begin{array}{l}3.18(2.01- \\
5.05)^{\star \star}\end{array}$ & $\begin{array}{l}3.42(2.12- \\
5.53)^{\star \star}\end{array}$ & NA & NA \\
\hline \multicolumn{5}{|l|}{ FoB } \\
\hline No & NA & NA & 1 & 1 \\
\hline Yes & NA & NA & $\begin{array}{l}3.18(2.01- \\
5.05)^{\star \star}\end{array}$ & $\begin{array}{l}3.23(2.01- \\
5.35)^{\star \star}\end{array}$ \\
\hline
\end{tabular}

$\mathbf{N A}=$ Not applicable to that particular category and not entered into the multivariable model as the univariable $p$ value was $\geq 0.200$.

${ }^{*} \mathrm{p}<0.005,{ }^{* *} \mathrm{p}<0.001$

Table 4: Predictors of having DS or FoB only or both. 


\begin{tabular}{lll}
$\begin{array}{l}\text { Predictors of having FoB } \\
\text { only }\end{array}$ & Predictors of having DS only & $\begin{array}{l}\text { Predictors of having both FoB } \\
\text { and DS }\end{array}$ \\
\hline $\begin{array}{l}\mathrm{N}=68 / 571 \\
(11 \%)\end{array}$ & $\mathrm{N}=86 / 589(14.6 \%)$ & $\begin{array}{l}\mathrm{N}=37 / 540 \\
(6.9 \%)\end{array}$ \\
\hline
\end{tabular}

Unadjusted

Adjusted Unadjusted Adjusted Unadjusted Adjusted

$\begin{array}{lllllll}\text { Variables } & \text { OR }(95 \% \mathrm{Cl}) & \begin{array}{l}\mathrm{OR} \\ (95 \% \mathrm{Cl})\end{array} & \mathrm{OR}(95 \% \mathrm{Cl}) & \begin{array}{l}\mathrm{OR} \\ (95 \% \mathrm{Cl})\end{array} & \text { OR }(95 \% \mathrm{Cl}) & \begin{array}{l}\text { OR } \\ (95 \% \mathrm{Cl})\end{array}\end{array}$

Age

\begin{tabular}{|c|c|c|c|c|c|c|}
\hline$£ 20$ years & 1 & 1 & 1 & 1 & 1 & 1 \\
\hline $\begin{array}{l}21-30 \\
\text { years }\end{array}$ & $\begin{array}{l}0.78(0.42- \\
1.43)\end{array}$ & $\begin{array}{l}0.69 \\
(0.33- \\
1.42)\end{array}$ & $1.66(0.87-3.17)$ & $\begin{array}{l}0.89 \\
(0.41- \\
1.94)\end{array}$ & $\begin{array}{l}0.89(0.38- \\
2.13)\end{array}$ & $\begin{array}{l}2.56(0.95- \\
6.89)\end{array}$ \\
\hline${ }^{3} 31$ years & $\begin{array}{l}0.55(0.26- \\
1.14)\end{array}$ & $\begin{array}{l}0.39 \\
(0.15- \\
1.04)\end{array}$ & $1.96(0.46-2.03)$ & $\begin{array}{l}0.59 \\
(0.22- \\
1.62)\end{array}$ & $\begin{array}{l}0.91(0.35- \\
2.33)\end{array}$ & $\begin{array}{l}6.29(1.43- \\
27.84)^{\star}\end{array}$ \\
\hline
\end{tabular}

Education level

\begin{tabular}{|c|c|c|c|c|c|c|}
\hline $\begin{array}{l}\text { No formal } \\
\text { education }\end{array}$ & 1 & 1 & 1 & 1 & 1 & 1 \\
\hline $\begin{array}{l}\text { Primary } \\
\text { education }\end{array}$ & $\begin{array}{l}1.04(0.55- \\
1.98)\end{array}$ & $\begin{array}{l}1.32 \\
(0.51- \\
3.45)\end{array}$ & $0.99(0.52-1.89)$ & $\begin{array}{l}0.36 \\
(0.16- \\
0.81) *\end{array}$ & $\begin{array}{l}1.25(0.53- \\
2.96)\end{array}$ & $\begin{array}{l}0.95(0.37- \\
2.43)\end{array}$ \\
\hline $\begin{array}{l}\text { Secondary } \\
\text { and higher }\end{array}$ & $\begin{array}{l}0.75(0.31- \\
1.83)\end{array}$ & $\begin{array}{l}1.33 \\
(0.61- \\
2.94)\end{array}$ & $2.43(1.21-4.87)^{*}$ & $\begin{array}{l}0.34 \\
(0.19- \\
0.63) \star\end{array}$ & $\begin{array}{l}0.50(0.13- \\
2.00)\end{array}$ & $\begin{array}{l}0.22(0.05- \\
0.99)^{*}\end{array}$ \\
\hline
\end{tabular}

\section{Parity}

\begin{tabular}{|c|c|c|c|c|c|c|}
\hline Nullipara & 1 & 1 & 1 & 1 & 1 & 1 \\
\hline Multipara & $\begin{array}{l}0.84(0.57- \\
1.58)\end{array}$ & $\begin{array}{l}1.42(0.69- \\
2.88)\end{array}$ & $\begin{array}{l}0.91 .02 \\
(0.57- \\
1.43)\end{array}$ & $\begin{array}{l}1.33(0.68- \\
2.60)\end{array}$ & $\begin{array}{l}0.51 \\
(0.26- \\
1.03)\end{array}$ & $0.27(0.09-0.87)^{*}$ \\
\hline
\end{tabular}

Income

\begin{tabular}{|c|c|c|c|c|c|c|}
\hline Adequate & 1 & 1 & 1 & 1 & 1 & 1 \\
\hline Inadequate & $\begin{array}{l}1.13(0.68- \\
1.88)\end{array}$ & NA & $2.06(1.26-3.36)^{*}$ & $\begin{array}{l}2.35 \\
(1.38- \\
3.99)^{*}\end{array}$ & $\begin{array}{l}2.11(1.02- \\
4.35)^{*}\end{array}$ & $\begin{array}{l}2.09(0.97- \\
4.52)\end{array}$ \\
\hline
\end{tabular}

Marital status

Married

1

1

1

1

1

1 


\begin{tabular}{|c|c|c|c|c|c|c|}
\hline Single & $\begin{array}{l}1.33(0.68- \\
2.59)\end{array}$ & NA & $2.04(1.27-3.29)^{\star}$ & $\begin{array}{l}1.98 \\
(1.18- \\
3.32)^{*}\end{array}$ & $\begin{array}{l}2.97(1.43- \\
6.18)\end{array}$ & $\begin{array}{l}2.57(1.14- \\
5.78)^{*}\end{array}$ \\
\hline \multicolumn{7}{|c|}{ Ever experienced obstetric complications } \\
\hline No & 1 & 1 & 1 & 1 & 1 & 1 \\
\hline Yes & $\begin{array}{l}1.15(0.69- \\
1.91)\end{array}$ & NA & $1.32(0.84-2.09)$ & NA & $\begin{array}{l}4.20(1.88- \\
9.37)^{\star}\end{array}$ & $\begin{array}{l}3.19(1.42- \\
7.19)^{*}\end{array}$ \\
\hline \multicolumn{7}{|c|}{ Planned pregnancy } \\
\hline No & 1 & 1 & 1 & 1 & 1 & 1 \\
\hline Yes & $\begin{array}{l}1.23(0.71- \\
2.13)\end{array}$ & NA & $1.58(0.93-2.67)$ & NA & $\begin{array}{l}1.06(0.52- \\
2.17)\end{array}$ & NA \\
\hline \multicolumn{7}{|c|}{ Previous perineal tear } \\
\hline No & 1 & 1 & 1 & & 1 & 1 \\
\hline Yes & $\begin{array}{l}1.49(0.80- \\
2.78)\end{array}$ & NA & $2.42(1.45-4.05) *$ & $\begin{array}{l}2.32 \\
(1.31- \\
4.08)^{*}\end{array}$ & $\begin{array}{l}1.46(0.64- \\
3.31)\end{array}$ & NA \\
\hline \multicolumn{7}{|c|}{ Social support from a male partner } \\
\hline No & 1 & 1 & 1 & 1 & 1 & 1 \\
\hline Yes & $\begin{array}{l}1.52(0.91- \\
2.52)\end{array}$ & NA & $1.59(1.00-2.51)^{\star}$ & $\begin{array}{l}1.46 \\
(0.87- \\
2.45)\end{array}$ & $\begin{array}{l}1.29(0.66- \\
2.52)\end{array}$ & NA \\
\hline
\end{tabular}

${ }^{*} p<0.005,{ }^{*} p<0.001 ; N A$, not applicable - indicates that the univariable $p$ value was $\geq 0.20$ and that the variable was not entered into the multivariable model.

Experience of obstetric complications in a previous pregnancy and birth was the strongest predictor of FoB. Another strong predictor of FoB was having a low educational level and lack of social support from a male partner in a previous childbirth. The strongest predictors of DS were being single, inadequate income, lack of social support from a male partner in a previous childbirth, having ever experienced obstetric complications, and have experienced a perineal tear before the index pregnancy (Table 3 ).

As shown in Table 4, the predictors of DS only, as compared with no FoB or DS were: lacking formal education; being unmarried; having inadequate income; having experienced a perineal tear in a previous delivery. The odds of having both FoB and DS as compared with no FoB and no DS were higher in older, single, and/or nulliparous women, those with a low education level, and those who had experienced obstetric complications.

\section{Discussion}

We aimed to determine the prevalence rates and predictors of FoB and DS among pregnant women attending antenatal clinics at selected public health facilities. We found that the prevalence rates of FoB and DS were in a 
similar range: $15.1 \%$ and $17.7 \%$, respectively. Some women had both FoB and DS, some had FoB but no DS or DS but no FoB, and others had neither FoB nor DS. Predictors of FoB and DS were lack of formal education, age above 30 years, never having given birth, being single, having had obstetric complications in the past, and having experienced a lack of male social support at a previous childbirth. Predictors of FoB were not having a formal education, while DS was seen in women without formal education, with inadequate income, who were single, and/or who had experienced a tear/episiotomy in a previous childbirth. Another key finding was that FoB and DS were strongly associated.

The prevalence rates of FoB and DS among Tanzania pregnant women in our study are within global ranges when the same validated tools are used for analysis $[10,14,15,17-19,47,52]$. The similar results across the globe might indicate that most women fear labour pain, being alone, and losing control of their body during labour and childbirth. Tanzanian women, like other women worldwide, have positive expectations on health facility childbirth. Most women who prefer health facility childbirth seek life-saving technology in case of obstetric complications. Some wish to have family members nearby to provide care and affection [53]. However, Tanzanian women's expectations may be hard to meet due to the high patient-health care provider ratio at most public health facilities. This could contribute to the high prevalence rates of FoB and DS. Also, disrespectful and abusive treatment from health providers during childbirth has been reported in Tanzania. For instance, women report being neglected/ignored, receiving physical and verbal abuse [54], being shouted at, threatened, slapped/pinched, or being left alone and forced to deliver by themselves [55]. All of these events could cause fear of childbirth.

Being above 30 years of age, not having a formal education or having primary education only, being single, being nulliparous, and having previous experience of obstetric complications were common among women with FoB in combination with DS. Surprisingly, we found that older women were more fearful and had more DS than younger ones. The findings were in line with those of a study done in Norway, assessing childbirth experiences in first-time mothers of advanced age [56]. The reason could be that older women have heard of more complications, which might intensify fear ahead of childbirth.

We found contradictory findings, showing that younger women/teenagers were more likely to develop DS and FoB than older women $[22,24,29]$. The discrepancy could be due to most teenagers being nulliparous with limited childbirth experience, which could manifest as DS and FoB. Health care providers should be attentive to all women and their differing childbirth expectations. FoB and DS were more likely in single women than married women. These findings were similar to those of previous studies $[13,16,28,43]$.

Further, women who had never given birth previously had a higher risk of having both FoB and DS. This was in line with other studies, which reported that nulliparous women presented with FoB more often than multiparous women $[6,25,27]$. However, we could not find any study assessing predictors of FoB in combination with DS for comparison.

Previous obstetric complications were strong predictors of both FoB and DS. Similar findings have been made in several other studies: a previous negative experience predicts FoB and DS in subsequent childbirth $[6,26,57,58]$. Negative birth experiences can lead to hesitance at becoming pregnant or giving birth in the future, resulting in delaying subsequent pregnancies or total avoidance of later pregnancies $[6-8,10,11,59,60]$. This might impact on women's future reproductive lives, likely diminishing trust in the ability to give birth and trust in maternity services. Such mistrust might lead to women not seeking maternity services from health facilities or opting for a $\mathrm{C} / \mathrm{S}$ rather than vaginal birth $[61,62]$. It is crucial to raise nurse-midwives awareness on the possible causes of 
negative birth experiences and to discuss how to support these women during subsequent pregnancies and childbirths. Women who have negative experiences should be identified during antenatal care for psychological support during pregnancy and childbirth. The same applies for women at risk of experiencing obstetric complications. These women will need to be empowered through provision of antenatal education on what to expect throughout the perinatal period.

In this study, women who received social support from their male partners during previous childbirths were less likely to report FoB and DS. This is in line with previous studies which reported that male partner support during childbirth is essential in alleviating fear related to childbirth [24,61] and preventing DS [63]. This highlights the importance of partner companionhip before, during, and after childbirth in a country where a male companion is not yet allowed or standard procedure during health facility childbirth.

Our study showed that FoB and DS were strongly associated. DS during pregnancy predicted having FoB. These findings are in line with findings from most systematic reviews and published studies $[59,64,65]$.

Depressive disorders are an important health problem globally. Among the participating women, DS was more likely to occur in women without formal education, who were single, who had an inadequate income and/or who had experienced tearing and/or an episiotomy in a previous delivery. Our findings were in line with previous studies showing that single mothers $[16,28,66]$ and those with inadequate income $[8,19,28]$ had increased risks of DS. Further, several previous studies have found that less educated people are more likely to be depressed than more educated ones $[28,67]$. There might be a pattern in how people of a similar educational background perceive the childbirth process, child upbringing, and associated resources. Another explanation could be that the more educated people are, the more they seek information from different sources, which could prevent them from emotional distress, manifesting as DS. Our findings were also consistent with another study showing that perineal wounds due to either tearing or episiotomy were associated with DS [47].

\section{Strengths And Limitations}

The study's major strength was the use of standardised, validated, and widely used tools for measuring FoB (WDEQ-A) and DS (EPDS), increasing credibility. The use of a visual aid scale was an added advantage that ensured clarity when using the W-DEQ Likert scale in the W-DEQ tool. Further, the sample was large enough to determine the prevalence rates and predictors of the outcome variables. Moreover, the quality of collected data was ensured as data collection was carried out under the direct supervision of the first author and general guidance from all team members. While many other similar studies have used self-administered tools, we used the intervieweradministered questionnaire technique, eliminating the hurdle for participants struggling to fill in responses on their own.

Using interviewers for data collection was a strength, but could also entail some limitations. If women adjusted their responses to please the interviewer, a social desirability bias might have occurred. This could lead to the prevalence rates of FoB and DS being slightly underestimated. Another limitation was that we did not know whether women in our study who were categorised as having FoB and DS had received any treatment. We were also unable to offer any support to women identified as having any problems. This is because the collected data were analysed and interpreted weeks later, meaning that women could not be referred for further management. Furthermore, the tools were only for screening purposes and therefore could not be used for clinical diagnosis. Our findings cannot be generalised to the first and second trimester of pregnancy, as data were collected during the 
third trimester. Comparisons with results from other studies may be impaired because of different data collection tools and cut-off points used to measure FoB and DS. However, we compared our findings with other studies that used only W-DEQ and EPDS tools to define FoB and DS [50] and DS [49], leaving out studies that used other tools to measure FoB and DS. W-DEQ-A might have been affected by being translated into Kiswahili, due to cultural diversity and differences in how women define and perceive childbirth in different countries. Furthermore, use of an adapted version of the EPDS validated in Kenya, where Kiswahili is also a national language, might be a limitation, as some words may have a different meaning when used in Tanzania.

\section{Conclusions}

Previous obstetric complications were the strongest predictor of FoB and DS, and FoB and DS were strongly associated with each other. Lack of social support from a male partner was also a predictor of FoB and DS. Not having a formal education was a predictor of FoB. Being single, having an inadequate income and having experienced a perineal tear were strong predictors of DS. Having FoB in combination with DS was more common in women aged above 30 years, without formal education, who were nulliparous and/or single. Understanding why some women are more prone to FoB and DS is vital in developing effective prevention and timely intervention to restore their mental health and psychological well-being throughout pregnancy, delivery, and after childbirth. This could, in turn, decrease mental suffering and negative consequences linked to FoB and DS.

Clinical implications from our results could be the possibilities for: a) proper screening of pregnant women for FoB and DS during the antenatal period; b) identifying and providing support to women at risk of developing mental illnesses and those who have experienced complications during previous pregnancies and childbirths.

\section{Abbreviations}

AOR: adjusted odds ratio; Cl: confidence interval: DS: depressive symptoms, EPDS: Edinburgh Postpartum Depression Scale; FoB: fear of childbirth; IQR: interquartile range; RAs: Research assistants; W-DEQ-A: Wijma Expectancy/Experience Delivery Questionnaire-A.

\section{Declarations}

\section{Ethical Approval and Consent to Participate}

Written permission was granted by Wijma, the author of W-DEQ-A, to use the tool in data collection in this study. Ethical clearance was sought from the Senate Research and Publication Committee of the Muhimbili University of Health and Allied Sciences with reference number 2018-03-09/AEC/Vol.XII/91. Permission to carry out the study was granted by the Regional Administrative Secretary in the Pwani region, Tanzania. We obtained written informed consent from the women who agreed to participate in the study. The study participants who were under 18 years of age were considered as emancipated minors and allowed to sign the consent form in the absence of their parents/guardians [68].

\section{Methodological consideration}

All methods were carried out in accordance with relevant guidelines and regulations.

\section{Consent to publication}

Page $18 / 23$ 
Not applicable

\section{Availability of data and materials}

The datasets used and/or analysed during the current study are available from the Directorof research and publication of Muhimbili Univeristy and Allied Sciences.

\section{Competing interests}

The authors declare they have no competing interests.

\section{Funding}

This research was funded by a Swedish international development agency (Sida) project, a collaboration between the Muhimbili University of Health and Allied Sciences in Tanzania and Uppsala University, Sweden. The project aimed at strengthening midwifery and obstetric services to improve maternal and newborn health in Tanzania.

\section{Authors' contribution}

AFM, ASS, ML, ABP, SL, and CM designed the study and selected the appropriate measurement tools. With inputs from all co-authors, AFM developed the study protocol, piloted the tools, trained the research assistants, supervised the data collection, and carried out data entry. AFM, ML, and ABP analysed the data. AFM developed the draft manuscript, and all authors contributed equally to refining the manuscript. All authors have read and approved the submitted manuscript.

\section{Acknowledgements}

We are thankful to the Pwani region administrative team for granting permission to carry out the study. Last but not least, we extend our sincere appreciation to the Statistician Mr. Patrik Ohagen, the assistant fieldwork supervisor, Ms. Dorkas Mwakawanga, the research assistants, the participants, and the health care providers of the health facilities who made this study possible.

\section{References}

1. Aktaş S, Aydın R. The analysis of negative birth experiences of mothers: a qualitative study. J Reprod Infant Psychol. 2019;37:176-92. doi:10.1080/02646838.2018.1540863.

2. Waldenström U, Hildingsson I, Rubertsson C, Rådestad I. A negative birth experience: Prevalence and risk factors in a national sample. Birth. 2004;31:17-27.

3. Waldenström U, Borg IM, Olsson B, Sköld M, Wall S. The Childbirth Experience: A Study of 295 New Mothers. Birth. 1996;23:144-53.

4. Bertucci V, Boffo M, Mannarini S, Serena A, Saccardi C, Cosmi E, et al. Assessing the perception of the childbirth experience in Italian women: A contribution to the adaptation of the childbirth perception questionnaire. Midwifery. 2012;28:265-74. doi:10.1016/j.midw.2011.02.009.

5. Smarandache A, Kim THM, Bohr Y, Tamim H. Predictors of a negative labour and birth experience based on a national survey of Canadian women. BMC Pregnancy Childbirth. 2016;16:1-9. doi:10.1186/s12884-0160903-2. 
6. Nilsson C, Lundgren I, Karlström A, Hildingsson I. Self reported fear of childbirth and its association with women's birth experience and mode of delivery: A longitudinal population-based study. Women and Birth. 2012.

7. Serçekuş P, Vardar O, Özkan S. Fear of childbirth among pregnant women and their partners in Turkey. Sex Reprod Healthc. 2020;24 February.

8. Wassif OM, Abdo AS, Elawady MA, Elmaksoud AEA, Eldesouky RS. Assessment of Postpartum Depression and Anxiety among Females Attending Primary Health Care Facilities in Qaliubeya Governorate, Egypt. 2019;2019.

9. Ryding EL, Lukasse M, Parys A-S Van, Wangel A-M, Karro H, Kristjansdottir H, et al. Fear of Childbirth and Risk of Cesarean Delivery: A Cohort Study in Six European Countries. Birth. 2015;42:48-55. doi:10.1111/birt.12147.

10. Klabbers GA. Severe Fear of Childbirth: Its Features, Assesment, Prevalence, Determinants, Consequences and Possible Treatments. 2016;25:107-27.

11. Hildingsson I, Haines $H$, Karlström A, Nystedt A. Presence and process of fear of birth during pregnancyFindings from a longitudinal cohort study. Women and Birth. 2017.

12. Nilsson C, Hessman E, Sjöblom H, Dencker A, Jangsten E, Mollberg M, et al. Definitions, measurements and prevalence of fear of childbirth: a systematic review. 2018;:1-15.

13. Onchonga D, MoghaddamHosseini V, Keraka M, Várnagy Á. Prevalence of fear of childbirth in a sample of gravida women in Kenya. Sex Reprod Healthc. 2020;24 January:100510. doi:10.1016/j.srhc.2020.100510.

14. Khwepeya M, Lee GT, Chen S, Kuo S. Childbirth fear and related factors among pregnant and postpartum women in Malawi. 2018;:1-10.

15. Underwood L, Waldie K, D'Souza S, Peterson ER, Morton S. A review of longitudinal studies on antenatal and postnatal depression. Archives of Women's Mental Health. 2016;19:711-20.

16. Thompson O, Ajayi I. Prevalence of Antenatal Depression and Associated Risk Factors among Pregnant Women Attending Antenatal Clinics in Abeokuta North Local Government Area, Nigeria. 2016. doi:10.1155/2016/4518979.

17. Manongi R, Rogathi J, Sigalla G, Mushi D, Rasch V, Gammeltoft T, et al. The Association Between Intimate Partner Violence and Signs of Depression During Pregnancy in Kilimanjaro Region , Northern. 2017.

18. Green EP, Tuli H, Kwobah E, Menya D, Chesire I, Schmidt C. Developing and validating a perinatal depression screening tool in Kenya blending Western criteria with local idioms: A mixed methods study. J Affect Disord. 2018;228 February 2017:49-59. doi:10.1016/j.jad.2017.11.027.

19. Rwakarema M, Premji SS, Nyanza EC, Riziki P, Palacios-Derflingher L. Antenatal depression is associated with pregnancy-related anxiety, partner relations, and wealth in women in Northern Tanzania: A cross-sectional study. BMC Womens Health. 2015;15:1-10.

20. Söderquist J. Posttraumatic stress after childbirth [Internet] [PhD dissertation]. [Linköping]: Linköpings universitet; 2002.

21. Saisto T, Salmela-Aro K, Nurmi JE, Halmesmäki E. Psychosocial characteristics of women and their partners fearing vaginal childbirth. Br J Obstet Gynaecol. 2001;108:492-8.

22. Spice K, Jones SL, Hadjistavropoulos HD, Kowalyk K, Stewart SH. Prenatal fear of childbirth and anxiety sensitivity. J Psychosom Obstet Gynecol. 2009;30:168-74. 
23. Fenwick J, Gamble J, Creedy JK, Buist DK, Ryding AL. Psycho-Social Pre-dictors of Childbirth Fear in Pregnant Women: An Australian Study. Open J Obstet Gynecol. 2014;4:531-43. doi:10.4236/ojog.2014.49075.

24. Gao L ling, Liu XJ, Fu BL, Xie W. Predictors of childbirth fear among pregnant Chinese women: A crosssectional questionnaire survey. Midwifery. 2015;31:865-70.

25. Rouhe H, Salmela-Aro K, Halmesmäki E, Saisto T. Fear of childbirth according to parity, gestational age, and obstetric history. BJOG An Int J Obstet Gynaecol. 2009;116:67-73.

26. Nilsson C, Lundgren I. Women's lived experience of fear of childbirth. Midwifery. 2007;25:e1-9.

27. Toohill J, Fenwick J, Gamble J, Creedy DK. Prevalence of childbirth fear in an Australian sample of pregnant women. BMC Pregnancy Childbirth. 2014;14:1-10.

28. Lancaster CA, Gold KJ, Flynn HA, Yoo H, Marcus SM, Davis MM. Risk factors for depressive symptoms during pregnancy: a systematic review. American Journal of Obstetrics and Gynecology. 2010;202:5-14.

29. Rich-Edwards JW, Kleinman K, Abrams A, Harlow BL, McLaughlin TJ, Joffe H, et al. Sociodemographic predictors of antenatal and postpartum depressive symptoms among women in a medical group practice. $\mathrm{J}$ Epidemiol Community Health. 2006;60:221-7.

30. Zeng Y, Cui Y, Li J. Prevalence and predictors of antenatal depressive symptoms among Chinese women in their third trimester: A cross-sectional survey. BMC Psychiatry. 2015;15.

31. Goyal D, Gay C, Lee KA. How much does Low Socioeconomic Status Increase the Risk of Prenatal and Postpartum Depressive Symptoms in First Time Mothers? Women's Heal Issues. 2010;20:96-104.

32. Kroll-Desrosiers AR, Crawford SL, Moore Simas TA, Clark MA, Bastian LA, Mattocks KM. Rates and Correlates of Depression Symptoms in a Sample of Pregnant Veterans Receiving Veterans Health Administration Care. Women's Heal Issues. 2019;29:333-40.

33. Taherifard P, Delpisheh A, Shirali R, Afkhamzadeh A, Veisani Y. Socioeconomic, Psychiatric and Materiality Determinants and Risk of Postpartum Depression in Border City of Ilam, Western Iran. Depress Res Treat. 2013;2013:e1-7.

34. Räisänen S, Lehto SM, Nielsen HS, Gissler M, Kramer MR, Heinonen S. Fear of childbirth in nulliparous and multiparous women: A population-based analysis of all singleton births in Finland in 1997-2010. BJOG An Int J Obstet Gynaecol. 2014;121:965-70.

35. Lukasse M, Schei B, Lena E, Study B. Prevalence and associated factors of fear of childbirth in six European countries. Sex Reprod Healthc. 2014;5:99-106. doi:10.1016/j.srhc.2014.06.007.

36. Laursen M, Hedegaard M, Johansen C. Fear of Childbirth: Predictors and Temporal Changes Among Nulliparous Women in the Danish National Birth Cohort. Obstet Anesth Dig. 2009;29:64-5.

37. Waldenström U, Hildingsson I, Ryding EL. Antenatal fear of childbirth and its association with subsequent caesarean section and experience of childbirth. BJOG An Int J Obstet Gynaecol. 2006;113:638-46.

38. Rouhe H, Salmela-Aro K, Gissler M, Halmesmäki E, Saisto T. Mental health problems common in women with fear of childbirth. BJOG An Int J Obstet Gynaecol. 2011;118:1104-11.

39. Nieminen K, Stephansson O, Ryding EL. Women's fear of childbirth and preference for cesarean section--a cross-sectional study at various stages of pregnancy in Sweden. Acta Obstet Gynecol Scand. 2009;88:80713.

40. Sydsjo G, Sydsjo A, Gunnervik C, Bladh M, Josefsson A. Obstetric outcome for women who received individualized treatment for fear of childbirth during pregnancy. Acta Obstet Gynecol Scand. 2012;91:44-9.

Page $21 / 23$ 
41. Aksoy M, Aksoy AN, Dostbil A, Celik MG, Ince I. The Relationship between Fear of Childbirth and Women's Knowledge about Painless Childbirth. Obstet Gynecol Int. 2014;2014:274303.

42. Adams SS, Eberhard-gran M, Eskild A. Fear of childbirth and duration of labour: a study of 2206 women with intended vaginal delivery. 2012;:15-8.

43. Heimstad R, Dahloe R, Laache I, Skogvoll E, Schei B. Fear of childbirth and history of abuse: Implications for pregnancy and delivery. Acta Obstet Gynecol Scand. 2006;85:435-40.

44. Slomian J, Honvo G, Emonts P, Reginster JY, Bruyère O. Consequences of maternal postpartum depression: $A$ systematic review of maternal and infant outcomes. Women's Health. 2019;15.

45. Holm-larsen CE, Madsen FK, Rogathi JJ, Manongi R, Mushi D, Meyrowitsch DW. Postpartum depression and child growth in Tanzania: a cohort study. 2018,:590-8.

46. Madeghe BA, Kimani VN, Vander Stoep A, Nicodimos S, Kumar M. Postpartum depression and infant feeding practices in a low income urban settlement in Nairobi-Kenya. BMC Res Notes. 2016;9:506-14.

47. Jha P, Larsson M, Christensson K, Svanberg AS. Fear of childbirth and depressive symptoms among postnatal women: A cross-sectional survey from Chhattisgarh , India. Women and Birth. 2018;31:e122-33. doi:10.1016/j.wombi.2017.07.003.

48. Wijma K, Wijma B, Zar M. Psychometric aspects of the W-DEQ; A new questionnaire for the measurement of fear of childbirth. J Psychosom Obstet Gynaecol. 1998;19:84-97.

49. Cox JL, Sagovsky JMHR. Detection of Postnatal Depression Development of the 10-item Edinburgh Postnatal Depression Scale. 1987;:782-6.

50. Zar M, Wijma K, Wijma B. Pre- and postpartum fear of childbirth in nulliparous and parous women. Scand J Behav Ther. 2001;30:75-84.

51. Massae AF, Larsson M, Leshabari S, Mbekenga C, Pembe AB, Svanberg AS. Fear of Childbirth: Validation of the Kiswahili Version of Wijma Delivery Expectancy/Experience Questionnaire Version A\&B in Tanzania. Submitted.

52. Chen $\mathrm{H}$, Chan $\mathrm{YH}$, Tan $\mathrm{KH}$, Lee T. Depressive symptomatology in pregnancy. A Singaporean perspective. Soc Psychiatry Psychiatr Epidemiol. 2004;39:975-9.

53. Shimpuku Y, Patil CL, Norr KF, Hill PD, Shimpuku Y, Patil CL, et al. Health Care for Women International Women ' s Perceptions of Childbirth Experience at a Hospital in Rural Tanzania Women 's Perceptions of Childbirth Experience at a Hospital in Rural Tanzania. Health Care Women Int. 2013;34:461-81.

54. Mselle LT, Moland KM, Mvungi A, Evjen-Olsen B, Kohi TW. Why give birth in health facility? Users' and providers' accounts of poor quality of birth care in Tanzania at Comprehensive Community Based Rehabilitation in Tanzania and Temeke hospitals in Dar es Salaam, and Mpwapwa district in Dodoma region. 2008. http://www.biomedcentral.com/1472-6963/13/174.

55. Kruk ME, Kujawski S, Mbaruku G, Ramsey K, Moyo W, Freedman LP. Disrespectful and abusive treatment during facility delivery in Tanzania: A facility and community survey. Health Policy Plan. 2018;33:e26-33.

56. Aasheim V, Waldenström U, Rasmussen S, Schytt E. Experience of childbirth in first-time mothers of advanced age - a Norwegian population-based study. BMC Pregnancy Childbirth. 2013;13.

57. Størksen HT, Garthus-Niegel S, Vangen S, Eberhard-Gran M. The impact of previous birth experiences on maternal fear of childbirth. Acta Obstet Gynecol Scand. 2013;92:318-24. 
58. Henriksen L, Grimsrud E, Schei B, Lukasse M. Factors related to a negative birth experience - A mixed methods study. Midwifery. 2017;51 January:33-9. doi:10.1016/j.midw.2017.05.004.

59. Dencker A, Nilsson C, Begley C, Jangsten E, Mollberg M, Patel H, et al. Causes and outcomes in studies of fear of childbirth: A systematic review. Women and Birth. 2019;32:99-111. doi:10.1016/J.WOMBI.2018.07.004.

60. Nilsson C, Bondas T, Lundgren I. Previous Birth Experience in Women With Intense Fear of Childbirth. JOGNN J Obstet Gynecol Neonatal Nurs. 2010;39:298-309.

61. Størksen HT, Garthus-Niegel S, Adams SS, Vangen S, Eberhard-Gran M. Fear of childbirth and elective caesarean section: A population-based study. BMC Pregnancy Childbirth. 2015;15:1-10. doi:10.1186/s12884015-0655-4.

62. Nilsson C, Bondas T, Lundgren I. Previous Birth Experience in Women With Intense Fear of Childbirth. JOGNN J Obstet Gynecol Neonatal Nurs. 2010;39:298-309.

63. Cankorur VS, Abas M, Berksun O, Stewart R. Social support and the incidence and persistence of depression between antenatal and postnatal examinations in Turkey: A cohort study. BMJ Open. 2015;5.

64. Räisänen S, Lehto SM, Nielsen HS, Gissler M, Kramer MR, Heinonen S. Fear of childbirth predicts postpartum depression: a population-based analysis of 511422 singleton births in Finland. 2013.

65. Dørheim SK, Bjorvatn B, Eberhard-Gran M. Can insomnia in pregnancy predict postpartum depression? A longitudinal, population-based study. PLoS One. 2014;9.

66. Shakeel N, Eberhard-Gran M, Sletner L, Slinning K, Martinsen EW, Holme I, et al. A prospective cohort study of depression in pregnancy, prevalence and risk factors in a multi-ethnic population. BMC Pregnancy Childbirth. 2015;15:1-11.

67. Hein A, Rauh C, Engel A, Häberle L, Dammer U, Voigt F, et al. Socioeconomic status and depression during and after pregnancy in the Franconian Maternal Health Evaluation Studies (FRAMES). Arch Gynecol Obstet. 2014;289:755-63.

68. Council for International Organizations of Medical Sciences (CIOMS) and World Health Organisation. International Ethical Guidelines for Health-related Research Involving Humans. 4th edition. Geneva; 2016. http://www.sciencedirect.com/science/article/B6VC6-45F5X02-9C/2/e44bc37a6e392634b1cf436105978f01.

\section{Supplementary Files}

This is a list of supplementary files associated with this preprint. Click to download.

- Additionalfile1.docx 